\title{
Quality Utility Modelling for Multimedia Applications for Android Mobile Devices
}

\author{
Ramona Trestian, Arghir-Nicolae Moldovan, Cristina Hava Muntean, Olga Ormond and Gabriel-Miro Muntean
}

\begin{abstract}
With the advances in mobile technologies, smart mobile computing devices have become increasingly affordable and powerful, leading to a significant growth in both the number of advanced mobile users and their bandwidth demands. Moreover multimedia streaming to these high-end mobile devices has become widespread. However, multimedia applications are known to be resource-hungry and in order to cope with this explosion of data traffic, operators have started deploying different, overlapping radio access network technologies. One important challenge in such a heterogeneous wireless environment is to ensure an Always Best Experience to the mobile user, anywhere and anytime. This paper proposes the Quality Utility, a realistic mapping function of the received bandwidth to user satisfaction for multimedia streaming applications. The Quality Utility is mapped to a Google Nexus One Android Mobile device and validated through objective and subjective tests.
\end{abstract}

Index Terms - multimedia applications, wireless networks, network selection

\section{INTRODUCTION}

$\mathrm{T}$ HE Always Best Connected (ABC) vision emphasis the scenario of a variety of radio access technologies that work together in order to form a global wireless infrastructure in which the end-users will benefit from an optimum service delivery via the most suitable available wireless network that satisfies their interests. The heterogeneous wireless environment, as illustrated in Figure 1, can be defined as a multi-technology multi-terminal multi-application and multiuser environment within which mobile users can roam freely. Advantages of such an environment include: it makes use of existing infrastructure, eliminating the cost of new technology deployments; it provides increased wireless capacity ensuring seamless mobility; it provides backward capability; it adds support for high data rates and low latency.

According to Cisco [1], by 2015 there will be over 7.1 billion mobile-connected devices, approximately equal to the world's population. Moreover, because of the growing popularity of video-sharing websites, the use of mobile video

Manuscript received April 27, 2012. The support of Enterprise Ireland, Science Foundation Ireland and Irish Research Council for Science, Engineering and Technology is gratefully acknowledged.

Quality Utility Modelling for Multimedia Applications for Android Mobile Devices

R. Trestian*, O. Ormond ${ }^{\ddagger}$, G-M. Muntean ${ }^{*}$ are with the Network Innovations Center ${ }^{t}$ and Performance Engineering Laboratory*, School of Electronic Engineering, Dublin City University, Dublin 9, Ireland (e-mails:\{ ramona, ormondo, munteang\}@eeng.dcu.ie).

A.-N. Moldovan and C. H. Muntean are with the National College of Ireland, Dublin 1, Ireland (e-mails: amoldovan@student.ncirl.ie and cmuntean@ncirl.ie). will more than double every year by 2015 , representing the highest growth rate of any application category. Thus, mobile video will represent two-thirds of the world's mobile data traffic by 2015.

In this context, the main challenge is to ensure an Always Best Experience to the mobile user, anywhere and anytime.

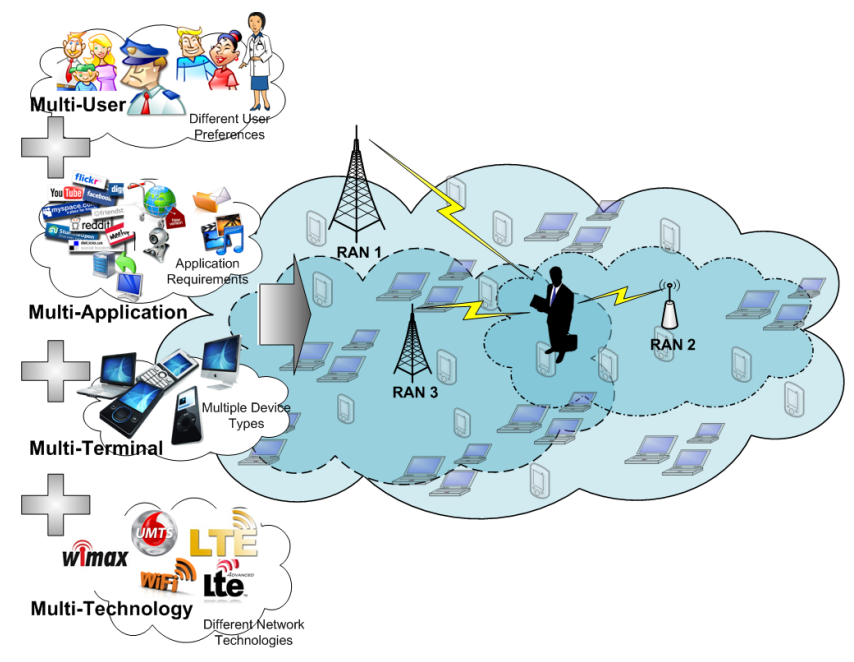

Figure 1. Heterogeneous Wireless Environment

In our previous work [2], a novel power-friendly access network selection mechanism is proposed. The mechanism makes use of a multiplicative multi-criteria weighted function in order to rank the available networks and select the least power consuming network. In order to do this, several utility functions are proposed focusing on energy, quality, cost, and mobility. This work proposes an enhanced quality utility that maps in a more realistic way the received bandwidth to user satisfaction for multimedia streaming applications. In order to assess the proposed solution, the quality utility is mapped to a Google Nexus One Android Mobile device and validated through objective and subjective tests.

The rest of the paper is structured as follows: section II summarizes the related work and section III describes the proposed quality utility. Section IV provides information related to the multimedia encoding whereas Section V details the video quality assessment. The modeling of the quality utility is presented in Section VI while the concluding remarks are given in Section VII. 


\section{RELATED WORKS}

In order to strengthen the Always Best Connected vision, various network selection mechanisms have been proposed in the research literature. Due to the different possible strategies and the numerous parameters involved in the process, researchers have tried many different techniques in order to find the most suitable network selection solution.

One of the first researchers to apply the SAW method in the area of network selection strategy was Wang et al. in 1999 [3]. They describe a policy-enabled handover system used to select the "best" wireless system at any moment. They define a score function as the cost of using a network at a certain time as a function of several parameters: the bandwidth it can offer, the power consumption of the network access, and the monetary cost of this network.

Since 1999 a number of other papers offering variations of this SAW method, have been produced, e.g., Adamopoulou et al. [4]. In order to scale different characteristics of different units to a comparable numerical representation, different normalized functions have been used, such as: exponential, logarithmic and linear piecewise functions [5]. One of the main drawbacks with SAW is that a poor value for one parameter can be heavily outweighed by a very good value for another parameter, so, for example, if a network has a low throughput, but a very good price, it may be selected over a slightly more expensive network with a much better throughput rate.

Bakmaz et al. [6] propose a network selection algorithm based on the TOPSIS method. The networks are ranked based on the closeness to the ideal solution using TOPSIS method. The proposed solution is evaluated using numerical examples. The parameters considered in the decision matrix are: available bandwidth, QoS level, security level, and cost. The results show that TOPSIS is sensitive to user preference and the parameter values.

In [5] Nguyen-Vuong et al. examine the disadvantages of previously proposed SAW algorithms and instead they propose the use of a weighted multiplicative method in the decision making mechanism. Their results show the inaccuracy of the SAW method and the benefits of using their proposed utility function together with a weighted multiplicative method.

An in-depth comparison study of the MADM methods is presented by Martínez-Morales et al. in [7]. The authors analyze the performance of SAW, TOPSIS, MEW, ELECTRE and GRA through simulations considering a $4 \mathrm{G}$ environment with three network types (e.g., WLAN, UMTS, and WiMAX) and six decision criteria (available bandwidth, total bandwidth, packet delay, packet jitter, packet loss, and monetary cost per byte. Their results show that SAW and TOPSIS are suitable for voice connections resulting in low jitter and packet delay, while GRA, MEW, and ELECTRE are suitable for data connections obtaining high throughput.

All these works look at network selection in general they do not focus on a particular application. But because of the continued growth of the video content, ensuring a seamless multimedia experience at high quality levels to the end-user has become a challenge. Thus, this leads to the necessity of defining multimedia-based solutions.

This paper proposes the modelling of a quality utility for multimedia streaming applications on an Android Mobile device, which can be used in the network selection mechanism in order to provide more realistic results.

\section{Quality UtiLITy}

The network selection score function makes use of the multiplicative exponential weighted (MEW) method as defined in equation (1) [2], and is based on four criteria: the energy consumption, the quality of the multimedia stream, the monetary cost, and the user mobility. The criteria can be divided into two classes: (1) the larger the better - higher values of the criteria are considered to be better than low values of the criteria (e.g., throughput); (2) the smaller the better - smaller values of the criteria are considered to be better than high values of the criteria (e.g., energy consumption, monetary cost). Because each criterion presents different ranges and units of measurement, they need to be scaled. The goal of the scaling process is to map all criteria onto non-dimensional values within the range $[0,1]$ to make them comparable. In order to do this, each criterion is scaled with the help of utility functions.

$$
U_{i}=u_{e_{i}}^{w_{e}} \cdot u_{q_{i}}^{w_{q}} \cdot u_{c_{i}}^{w_{c}} \cdot u_{m_{i}}^{w_{m}}
$$

where: $U_{i}$ - overall score function for RAN $i ; u_{e}, u_{q}, u_{c}$, and $u_{m}$ are the utility functions defined for energy, quality in terms of received bandwidth, monetary cost for RAN $i$, and user mobility respectively. Also $w_{e}+w_{q}+w_{c}+w_{m}=1$, where $w_{e}$, $w_{q}, w_{c}$, and $w_{m}$ are the weights for the considered criteria, representing the importance of a parameter in the decision algorithm.

In order to map the received bandwidth to user satisfaction for multimedia streaming applications in a more realistic way, a zone-based sigmoid quality utility function is defined, and illustrated in Figure 2. The utility is computed based on: the minimum throughput $\left(\mathrm{Th}_{\min }\right)$ needed to maintain the multimedia service at a minimum acceptable quality (values below this threshold result in unacceptable quality levels i.e., zero utility), the required throughput $\left(\mathrm{Th}_{\mathrm{req}}\right)$ in order to ensure high quality levels for the multimedia service; the maximum throughput $\left(\mathrm{Th}_{\max }\right)$, values above this threshold result in quality levels which are higher than most human viewers can distinguish between and so anything above this maximum threshold is a waste. The mathematical formulation of this quality utility function is given in equation (2) where: $\alpha$ and $\beta$ are two positive parameters which determine the shape of the utility function (no unit), Th is the predicted average throughput for each of the candidate networks (Mbps), $\mathrm{Th}_{\min }$ is the minimum throughput (Mbps), and $\mathrm{Th}_{\max }$ is the maximum throughput (Mbps). The quality utility has values in the $[0,1]$ interval and no unit. In order to determine the exact shape of the utility function the values of $\alpha$ and $\beta$ need to be calculated. For this, two equations are needed. 
$u_{q}(T h)=\left\{\begin{array}{ccc}0 & , & T h<T h_{\min } \\ 1-e^{\frac{-\alpha * T h^{2}}{\beta+T h}} & , & T h_{\min }<=T h<T h_{\max } \\ 1 & , & \text { otherwise }\end{array}\right.$

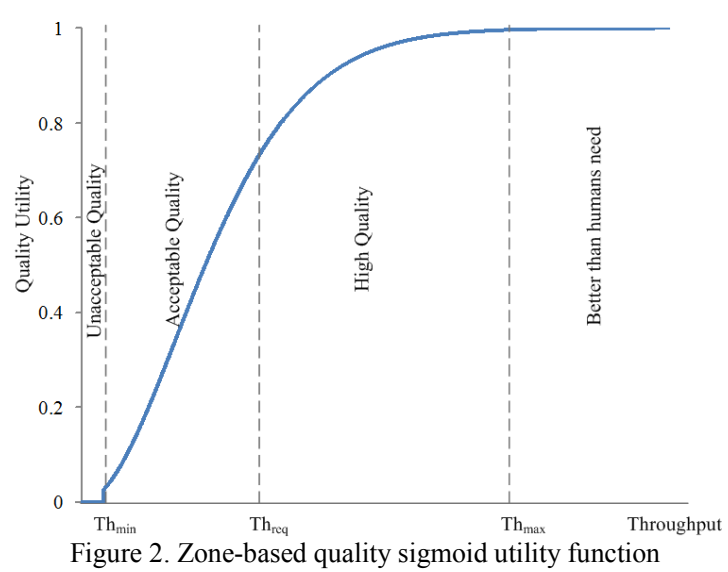

The first equation can be obtained from knowing that when the throughput reaches $\mathrm{Th}_{\max }$ the corresponding utility $u$ will be equal to $u_{\max }$. Thus, the first equation is defined as follows:

$$
1-e^{\frac{-\alpha * T h_{\max }^{2}}{\beta+T h_{\max }}}=u_{\max }
$$

From equation (4.5) a relationship between $\alpha$ and $\beta$ can be obtained as follows:

$$
\alpha=\frac{\ln \left(1-u_{\text {max }}\right)\left(\beta+T h_{\text {max }}\right)}{-T h_{\text {max }}^{2}}
$$

Now that the relationship between $\alpha$ and $\beta$ is defined, a second equation is needed in order to calculate their values. The required throughput, $\mathrm{Th}_{\text {req }}$, illustrated in Figure 2 can be defined mathematically as the throughput before which the utility function is convex and after which the utility becomes concave. This means that the second-order derivative of the utility function is zero at this point. After computing the second-order derivate and replacing $\alpha$ with equation (4), equation (5) is obtained. The positive solution of equation (4.7) represents the value of $\beta$. The quality utility is further validated in the next Sections.

$$
\begin{aligned}
& {\left[1+2 \ln \left(1-u_{\max }\right)\left(\frac{T h_{r e q}}{T h_{\max }}\right)^{2}\right] \beta^{3}+\left[T h_{r e q}+2 \ln \left(1-u_{\max }\right) \frac{T h_{r e q}^{2}}{T h_{\max }}\right.} \\
& \left.+2 \ln \left(1-u_{\max }\right) \frac{T h_{r e q}^{3}}{T h_{\max }^{2}}\right] \beta^{2}+\left[2 \ln \left(1-u_{\max }\right)\right. \\
& \left.\frac{T h_{r e q}^{3}}{T h_{\max }}+\ln \left(1-u_{\max }\right) \frac{T h_{r e q}^{4}}{2 T h_{\max }^{2}}\right] \beta \\
& +\ln \left(1-u_{\max }\right) \frac{T h_{r e q}^{4}}{2 T h_{\max }}=0
\end{aligned}
$$

\section{Multimedia ENCODING}

The Blender Foundation's 10 minute long Big Buck Bunny ${ }^{1}$ animated clip was used for validating the quality utility. A high quality version of the clip was transcoded at five different

${ }^{1}$ Big Buck Bunny Clip http://www.bigbuckbunny.org/. quality levels, following the guidelines for encoding clips for

\begin{tabular}{|c|c|c|c|c|c|}
\hline \multirow[b]{2}{*}{$\begin{array}{l}\text { Quality } \\
\text { Level }\end{array}$} & \multicolumn{5}{|c|}{ Encoding Parameters } \\
\hline & $\begin{array}{l}\text { Video } \\
\text { Codec }\end{array}$ & $\begin{array}{l}\text { Overall } \\
\text { Bitrate } \\
\text { [Kbps] } \\
\end{array}$ & $\begin{array}{l}\text { Resolution } \\
\text { [pixels] }\end{array}$ & $\begin{array}{c}\text { Frame } \\
\text { Rate } \\
\text { [fps] } \\
\end{array}$ & $\begin{array}{l}\text { Audio } \\
\text { Codec }\end{array}$ \\
\hline QL1 & \multirow{5}{*}{$\begin{array}{c}\text { H.264/ } \\
\text { MPEG- } \\
4 \\
\text { AVC } \\
\text { Baseline } \\
\text { Profile }\end{array}$} & 1920 & $800 \times 448$ & 30 & \multirow{5}{*}{$\begin{array}{c}\text { AAC } \\
25 \\
\mathrm{Kbps} \\
8 \mathrm{KHz}\end{array}$} \\
\hline QL2 & & 960 & $512 \times 288$ & 25 & \\
\hline QL3 & & 480 & $320 \times 176$ & 20 & \\
\hline QL4 & & 240 & $320 \times 176$ & 15 & \\
\hline QL5 & & 120 & $320 \times 176$ & 10 & \\
\hline
\end{tabular}
multi-bitrate adaptive streaming ${ }^{2}$. The encoding characteristics of the five test sequences are presented in Table 1. H.264/MPEG-4 AVC video compression and AAC audio compression were used together with MP4 container ${ }^{3}$.

TABLE 1. Encoding Settings for the Multimedia Test Sequences

The encoded resolution was varied together with the bitrate in order to maintain a consistent level of compression quality. Figure 3 illustrates an example of variable resolution encoding. For example, Figure 3a presents a high quality level encoded at $800 \times 448$ and $1920 \mathrm{kbps}$. If the same resolution is kept and the bit rate only is lowered as in Figure $3 \mathrm{~b}$, some compression side effects can be noticed, such as: blockiness, color smearing, twirling details, etc. On the other side, by lowering the resolution together with the bitrate as shown in Figure $3 \mathrm{c}$ the blockiness effect is not visible, the picture presents some blurry aspects but the quality is relatively good.

Considering these aspects, the highest resolution was selected as $800 \times 448$ pixels to fit the screen resolution of the Android Nexus One device (800x480 pixels), while maintaining the original aspect ratio of the multimedia clip (16:9). The smallest resolution was selected as 320x176 and was kept the same for the last three quality levels. Going below this value, the risk of providing bad quality to the user appears, as very small video can result in bad full-screen experience. The video frame rate was also step-wise decreased from 30fps for QL1 to 10fps for QL5. The overall bitrate was decreased by half between consecutive quality levels from $1920 \mathrm{Kbps}$ (QL1) to $120 \mathrm{Kbps}$ (QL5). Out of these overall bitrate values, $25 \mathrm{Kbps}$ corresponded to the audio stream while the rest corresponds to video stream. The audio component was not varied for the different quality levels.

\section{Multimedia Quality ASSESSMENT}

\section{A. Objective Quality Assessment}

One of the important aspects of the multimedia delivery is the user perceived quality of the multimedia stream. There are two methods which can be used in order to assess the video quality: objective and subjective methods. The most widely used objective metric is the full-reference Peak Signal-toNoise Ratio (PSNR). In this paper the MSU Video Quality

\footnotetext{
${ }^{2}$ Smooth Streaming Multi-Bitrate Calculator http://alexzambelli.com/W MV/MBRCalc.html

${ }^{3}$ MP4 multimedia container format http://en.wikipedia.org/wiki/MPEG 4_Part_14
} 


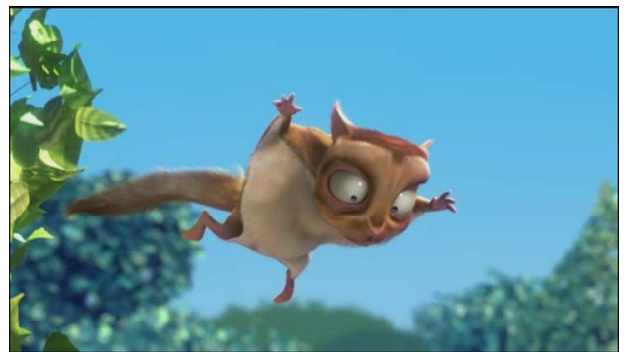

a) encoding at $800 \times 448 / 1920 \mathrm{kbps}$

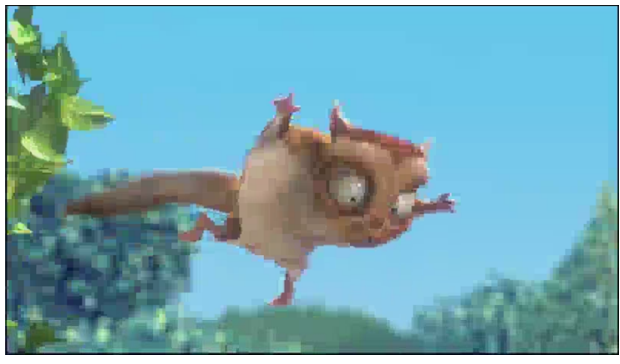

b) encoding at $800 \times 448 / 120 \mathrm{kbps}$

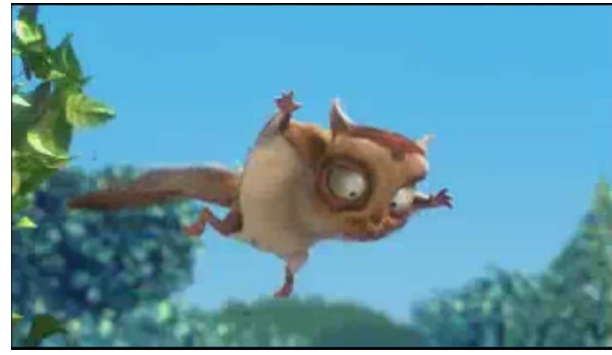

c) encoding at $320 \times 176 / 120 \mathrm{kbps}$

Figure 3. Variable Resolution Encoding - Example

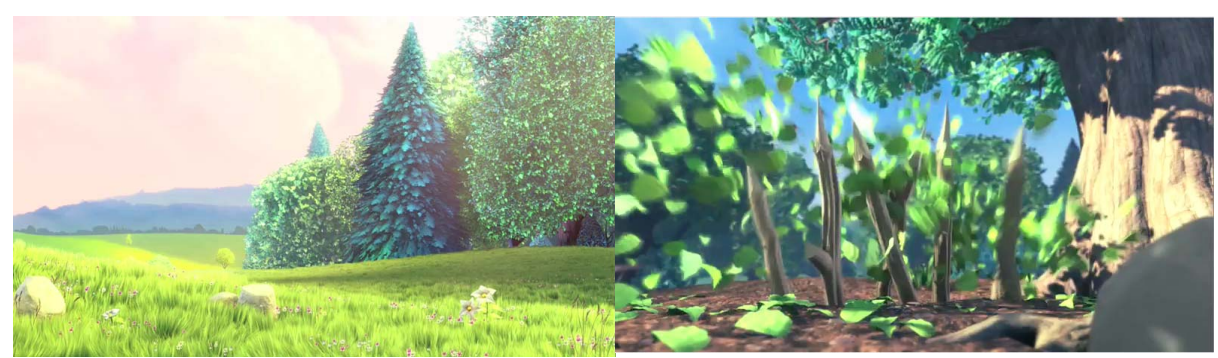

a) Sequence $\mathrm{A}$

b) Sequence B

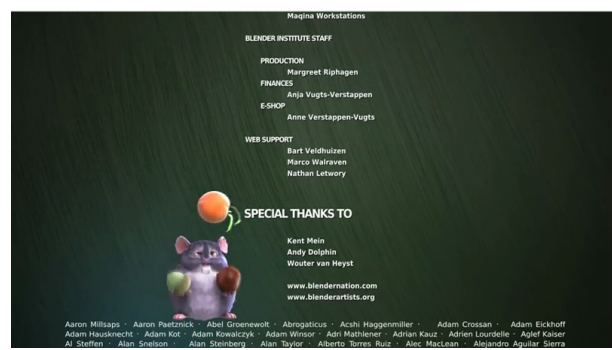

c) Sequence C b)

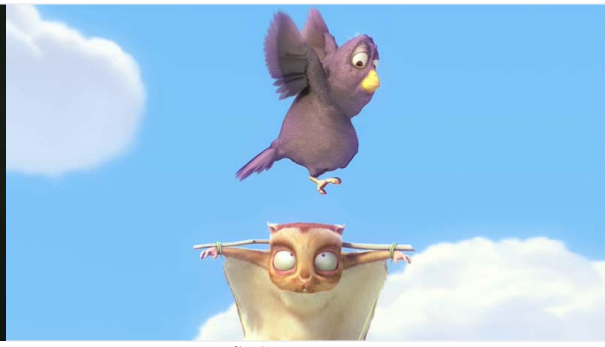

d) Sequence D

Figure 4. Test sequences used for the subjective study

Measurement Tool $^{4}$ software was used for computing the objective PSNR values in order to estimate the human perceived visual quality offered by the five encoding settings used. PSNR values are computed by comparing, on a pixel-bypixel basis, the quality of the degraded versions (QL2 to QL5) with regard to that of the highest quality sequence (QL1). In order to do this, all the clips were scaled to the same video resolution and video frame rate. Although employing the scaling process is not ideal, by computing PSNR, one gets a good idea of the human perceived quality levels for these video sequences.

${ }^{4}$ MSU Video Quality Measurement Tool - http://compression.ru/video/ quality_measure/video_measurement_tool_en.html

\section{B. Subjective Quality Assessment}

\section{Subjective Tests - Setup}

Using only objective metrics to assess how human subjects perceive the quality of the five levels of the multimedia stream, is not enough as the objective scores do not always correlate with the subjective scores. Hence a subjective study was also conducted [8]. For this purpose, four 20 seconds long test sequences with different spatial and temporal characteristics were extracted from the original 10 minute long multimedia clip of each quality level. A total number of 20 test sequences were used for the subjective study. Representative frames of the four sequences are illustrated in Figure 4.

Sequence A was selected so that it presents a medium level of spatial information and a low level of temporal information. Thus, in this clip (Figure 4a), the camera slowly pans over a 
natural landscape scene. Sequence B (Figure 4b) presents the highest level of spatial and temporal complexity making it the most complex to encode. It presents fast changing scenes with dynamic elements as well as characters.

Sequence C (Figure 4c) presents high spatial information but low temporal complexity. The small moving details, represented by the closing credits, make the clip difficult to encode at good quality low resolutions. Sequence D (Figure 34) has the lowest of spatial information. The video presents two characters, from which only one is slowly moving across the scene, on a static background.

All the test sequences were played locally in full screen on the Android Nexus One device and they were displayed randomly in order to minimize the order effect. Similar testing conditions were maintained for all the participants and standard recommendations for assessing the visual quality of the multimedia applications were followed according to ITU-T P.910 [9]. The Absolute Category Rating (ACR) [9] method was used, and the subjects had to individually rate the quality of each sequence on a 5-point scale (e.g., 1 - Bad, 2 - Poor, 3 Fair, 4 - Good, 5 - Excellent). A total number of 16 (Males = 10 , Females $=6$ ) non-expert subjects with ages between 22 and 45 years old (Average Age $(\mathrm{AVG})=28$, standard deviation $(\mathrm{STDEV})=6$ ) have participated in the study. All the subjects have reported that they had normal vision or have corrected to normal vision (they were wearing glasses).

\section{Subjective Tests - Results}

The results of the subjective study are illustrated in Figure 5 [8]. For each sequence of the 20 test sequences, the mean value represented by the Mean Opinion Score (MOS), and the standard deviation (STDEV) of the statistical distribution of the assessment grades were computed. The objective PSNR and the subjective MOS results are listed in Table 2 together with the perceived quality and impairment mapping.

TABLE 2. OBJECTIVE AND SUBJECTIVE RESULtS

\begin{tabular}{|c|c|c|c|c|}
\hline $\begin{array}{l}\text { Quality } \\
\text { Level }\end{array}$ & $\begin{array}{l}\text { PSNR } \\
\text { [dB] }\end{array}$ & $\begin{array}{l}\text { Subjective } \\
\text { MOS }\end{array}$ & $\begin{array}{c}\text { Perceived } \\
\text { Quality }\end{array}$ & Impairment \\
\hline QL1 & - & 4.84 & Excellent & Imperceptible \\
\hline QL2 & 47 & 4.63 & Excellent & Imperceptible \\
\hline QL3 & 41 & 4.33 & Good & $\begin{array}{c}\text { Perceptible but } \\
\text { not annoying }\end{array}$ \\
\hline QL4 & 36 & 3.70 & Good & $\begin{array}{l}\text { Perceptible but } \\
\text { not annoying }\end{array}$ \\
\hline QL5 & 31 & 3.38 & Fair & $\begin{array}{c}\text { Slightly } \\
\text { annoying }\end{array}$ \\
\hline
\end{tabular}

Looking at the average values in Figure $5 \mathrm{a}$, all the sequences corresponding to QL1-QL3 scored above 4 (Good), with eight of them scoring above 4.5 (Excellent). Four out of the eight test sequences corresponding to QL4 and QL5, scored above 3.5 (Good), while the other four below 3.5 but above 2.5 (Fair). Looking across the four test sequences, two quality levels scored Excellent (e.g., QL1 and QL2 with the avg. MOS 4.84 and 4.63, respectively), two scored Good (e.g., QL3 and QL4 with the avg. MOS 4.33 and 3.70, respectively) and one Fair (e.g., QL5 with the avg. MOS 3.38). Looking at the average standard deviation values, as illustrated in Figure $5 \mathrm{~b}$, it can be noticed that the STDEV MOS is increasing as the video quality decreases (e.g., MOS STDEV for QL1 $=0.35$ whereas MOS STDEV for QL5 $=0.90$ ). The Pearson correlation further indicates that there is decreasing relationship between the MOS and STDEV values $(\mathrm{r}=$ 0.846), thus the ratings across participants tend to have a higher variation, for the clips with lower perceived quality.

\section{Modeling THE Quality UTILITY}

This section shows the validation for the choice of the quality utility which can be integrated in the network selection mechanism as described in Section III for more realistic results. The quality utility is modeled as a sigmoid function, based on the idea that there is a minimum throughput required by a multimedia application in order to provide a minimum acceptable quality to the user. If the received throughput goes below this value, the quality becomes unacceptable and the quality utility is zero, meaning that the provided service is worth nothing to the user. On the other side, there is a maximum throughput required by a multimedia application in order to provide high quality levels to the user. The received throughput that goes above this maximum will not add much to the already existing high quality, but still it will increase the energy consumption and possibly it is wasted traffic on the operators' network.

In this paper five different quality levels are considered, from QL1 (high quality) to QL5 (low quality). The five quality levels were chosen as described in Section IV and their characteristics were illustrated in Table 1. After performing the subjective tests, described in Section V, a Mean Opinion Score (MOS) was assigned to each quality level. Figure 6 shows the relationship between the quality utility, received throughput (Quality Levels) and MOS.

As it can be noticed from Figure 5, the results obtained through subjective testing for the five quality levels, validate the choice of the sigmoid function.

Based on the quality levels' characteristics, the quality utility is modeled as in equation (6.1).

$$
u_{q}(T h)=\left\{\begin{array}{ccc}
0 & , & T h<0.120 \\
1-e^{\frac{-\alpha * T h^{2}}{\beta+T h}} & , & 0.120<=T h<1.920 \\
1 & , & \text { otherwise }
\end{array}\right.
$$

where $\alpha$ and $\beta$ are two positive parameters that are determined knowing that: (1) for $\mathrm{Th}_{\max }$ (1.920Mbps) the utility has its maximum value (e.g., $\mathrm{u}_{\max }=0.99$ in order to avoid $\ln (0)$ which is invalid); (2) the second order derivate of $u_{q}$ equals 0 for $\mathrm{Th}_{\mathrm{req}}(0.480 \mathrm{Mbps})$. The two conditions mentioned above will reduce to equation (7) and (8). 

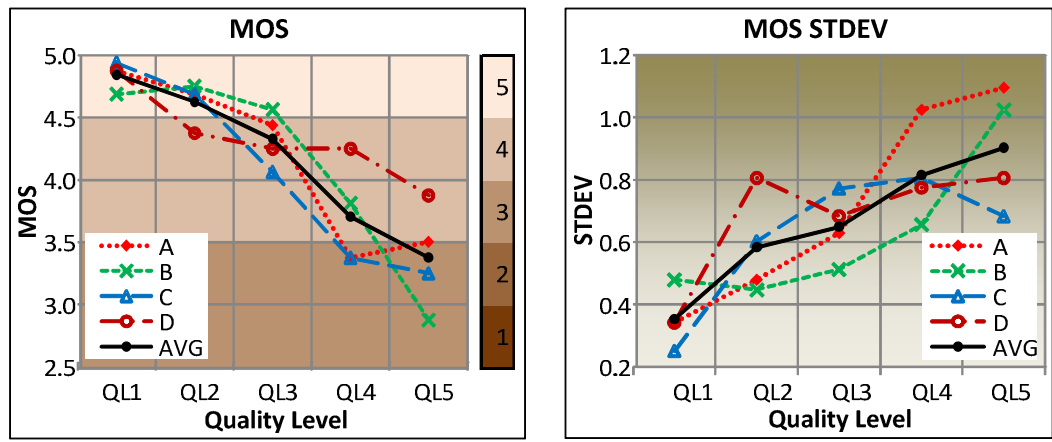

\begin{tabular}{|l|l|l|l|l|l|}
\hline Sequence & \multicolumn{1}{|c|}{ QL1 } & \multicolumn{1}{|c|}{ QL2 } & QL3 & QL4 & QL5 \\
\hline A & 4.88 & 4.69 & 4.44 & 3.38 & 3.50 \\
\hline B & 4.69 & 4.75 & 4.56 & 3.81 & 2.88 \\
\hline C & 4.94 & 4.69 & 4.06 & 3.38 & 3.25 \\
\hline D & 4.88 & 4.38 & 4.25 & 4.25 & 3.88 \\
\hline AVG & 4.84 & 4.63 & 4.33 & 3.70 & 3.38 \\
\hline
\end{tabular}

a) MOS

\begin{tabular}{|l|c|c|c|c|c|}
\hline Sequence & QL1 & QL2 & QL3 & QL4 & QL5 \\
\hline A & 0.34 & 0.48 & 0.63 & 1.02 & 1.10 \\
\hline B & 0.48 & 0.45 & 0.51 & 0.66 & 1.02 \\
\hline C & 0.25 & 0.60 & 0.77 & 0.81 & 0.68 \\
\hline D & 0.34 & 0.81 & 0.68 & 0.77 & 0.81 \\
\hline AVG & 0.35 & 0.58 & 0.65 & 0.82 & 0.90 \\
\hline
\end{tabular}

b) MOS STDEV

Figure 5. Results of the Subjective Quality Assessment [8]

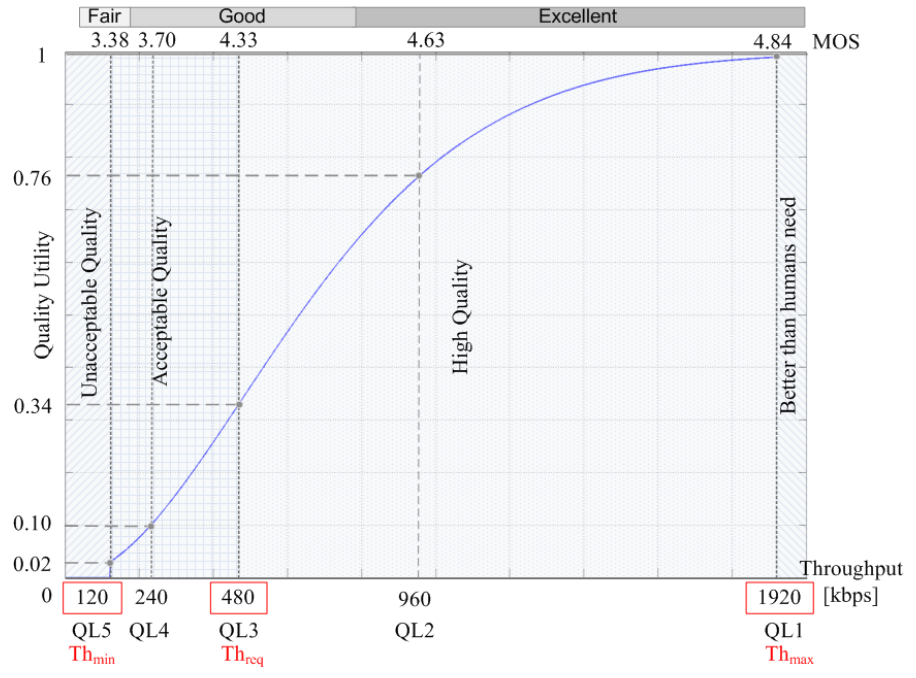

Figure 6. Quality Utility - Validation

$$
\begin{aligned}
& \alpha=\frac{\ln \left(1-u_{\text {max }}\right)\left(\beta+T h_{\text {max }}\right)}{-T h_{\text {max }}^{2}}=5.72 \\
& {\left[1+2 \ln \left(1-u_{\text {max }}\right)\left(\frac{T h_{r e q}}{T h_{\text {max }}}\right)^{2}\right] \beta^{3}+\left[T h_{r e q}+2 \ln \left(1-u_{\text {max }}\right) \frac{T h_{r e q}^{2}}{T h_{\text {max }}}\right.} \\
& \left.+2 \ln \left(1-u_{\text {max }}\right) \frac{T h_{r e q}^{3}}{T h_{\text {max }}^{2}}\right] \beta^{2}+\left[2 \ln \left(1-u_{\text {max }}\right)\right. \\
& \left.\frac{T h_{r e q}^{3}}{T h_{\text {max }}^{3}}+\ln \left(1-u_{\text {max }}\right) \frac{T h_{r e q}^{4}}{2 T h_{\text {max }}^{2}}\right] \beta \\
& +\ln \left(1-u_{\text {max }}\right) \frac{T h_{r e q}^{4}}{2 T h_{\text {max }}}=0 \\
& \Rightarrow \beta=2.66
\end{aligned}
$$

where the positive solution of equation (8) is the value of $\beta$. In this particular case the values for $\alpha$ and $\beta$, after solving all the mathematical computations, are 5.72 and 2.66, respectively. For any other choice of quality levels, the procedure of identifying the parameters of the quality utility function is similar.

\section{CONCLUSIONS}

Nowadays the network operators consider that if they offer individual high throughput this is translated into satisfied users. However, the excellent perceived quality of service does not always results from high throughput, and a good trade-off between quality and other parameters (e.g., energy conservation) is needed in order to keep the user satisfied. However, in order to offer a good trade-off the quality has to be mapped to the user satisfaction in the most realistic way possible. This paper proposes a quality utility model for multimedia streaming applications that maps the received bandwidth (quality level) to the user satisfaction. The modeling was done based on the results obtained from the subjective tests for video quality assessment.

\section{REFERENCES}

[1] Cisco Systems, Inc., "Cisco Visual Networking Index: Global Mobile Data Traffic Forecast Update, 2010-2015", 2011.

[2] R. Trestian, O. Ormond and G-M. Muntean, "Power-Friendly Access Network Selection Strategy for Heterogeneous Wireless Multimedia Networks", IEEE Int. Symp. on Broadband Multimedia Systems and Broadcasting, BMSB 2010.

[3] H.J. Wang, R.H. Katz, and J. Giese, "Policy-enabled handoffs across heterogeneous wireless networks", IEEE Workshop on Mobile Computing Systems and Applications, WMCSA 1999.

[4] E. Adamopoulou, "Intelligent Access Network Selection in Heterogeneous Networks - Simulation Results", Int. Symp. on Wireless Communication Systems, 2005.

[5] Q.-T. Nguyen-Vuong, Y. Ghamri-Doudane, N. Agoulmine, "On utility models for access network selection in wireless heterogeneous networks" IEEE Network Operations \& Manag. Symp. NOMS 2008.

[6] B. Bakmaz, Z. Bojkovic, and M. Bakmaz, "Network Selection Algorithm for Heterogeneous Wireless Environment," IEEE Int. Symp. on Personal, Indoor \& Mobile Radio Comms., PIMRC 2007.

[7] J. D. Martínez-Morales, U. Pineda-Rico, and E. Stevens-Navarro, "Performance comparison between MADM algorithms for vertical handoff in 4G networks", Int. Conf. on Electrical Engineering Computing Science and Automatic Control, CCE 2010.

[8] R. Trestian, A-N. Moldovan, O. Ormond, and G-M. Muntean, "Energy Consumption Analysis of Video Streaming to Android Mobile Devices", IEEE/IFIP Network Operations \& Management Symp., NOMS 2012

[9] ITU-T, P.910, "Subjective video quality assessment methods for multimedia applications" International Telecommunication Union, 2008. 